\title{
Neo Formación Ósea de Rama y Cuerpo Mandibular Tras Uso de Injerto No Vascularizado de Cresta llíaca en Re- construcción Inmediata tras Resección de Ameloblastoma Folicular en Paciente de 11 años. Caso Clínico
}

\author{
Neo Bone Formation of the Mandibular Branch and Body After the Use of a Non- \\ Vascularized Iliac Crest Graft in Immediate Reconstruction after Resection of \\ Follicular Ameloblastoma in an 11-Year-Old Patient. Clinical Case
}

Pedro Tapia Contreras'; Pablo Arrué Delgado²; Sebastián Mordoh Cucurella3; Christopher Reyes Araya ${ }^{4}$ \& Benjamín Rosenberg Dueñas ${ }^{5}$

TAPIA, C. P.; ARRUÉ, D. P.; MORDOH, C. S.; REYES, A. C. \& ROSENBERG, D. B. Neo formación ósea de rama y cuerpo mandibular tras uso de injerto no vascularizado de cresta ilíaca en reconstrucción inmediata tras resección de Ameloblastoma Folicular en paciente de 11 años. Caso clínico. Int. J. Odontostomat., 15(3):569-573, 2021.

RESUMEN: El tratamiento "gold standar" para los ameloblastomas mandibulares agresivos, especialmente en aquellas lesiones cuyos márgenes de exceresis superan los $6 \mathrm{~cm}$, es la resección y reconstrucción inmediata con injerto óseo autólogo microvascularizado. Esto se basa en la necesidad de aporte sanguíneo para extensiones amplias de hueso implantado. Es necesario en estos casos una correcta estabilización y fijación del injerto mediante placas de reconstrucción (de titanio),y la ausencia de tejido sometido a radioterapia en la zona a tratar; siendo estos últimos elementos de gran importancia para el éxito quirúrgico. En el caso de pacientes pediátricos, los procesos de oseointegración y reparación ósea se ven beneficiados por el crecimiento fisiológico que conlleva la presencia de multiples factores de crecimiento. El objetivo de este reporte de caso es presentar el tratamiento de un ameloblastoma mandibular en paciente pediátrico tratado mediante resección y reconstrucción con injerto de cresta ilíaca no vascularizado de $10 \mathrm{~cm}$ de amplitud. Logrando neoformación ósea 3D del area reconstruida.

PALABRAS CLAVE: pediatría, injerto óseo autógeno no vascularizado, reconstrucción de mandíbula.

\section{INTRODUCCIÓN}

Los defectos óseos mandibulares se definen como la pérdida de un segmento estructural de la mandíbula, esto puede ocurrir por diferentes causas como trauma, quistes, tumores, osteomelitis crónica, osteoradionecrosis y osteonecrosis por medicamentos (Akinbami, 2016). Cuando este defecto compromete el tejido óseo alveolar y el hueso basal mandibular con un gap igual o mayor a $2 \mathrm{~cm}$, puede existir desinserciones musculares, alteraciones: estéticas, funcionales, sensitivas, dificultad en las funciones masticatorias y del habla (Schlieve et al., 2015). El tratamiento gold estándar para grandes defectos es el injerto óseo autógeno vascularizado, que permite la irrigación inmediata del tejido óseo y blando circundante asegurando la vitalidad del tejido injertado (Okoturo, 2016). El propósito de una reconstrucción es devolver al paciente las características anatómicas, funcionales, biomecánicas y estéticas, por lo cual la reconstrucción de los defectos óseos propone un gran desafío para el Cirujano Maxilofacial.

${ }^{1}$ Servicio Cirugía Máxilofacial Clínica Red Salud Vitacura, Santiago, Chile. Hospital Regional de Rancagua, Chile.

2 DDS, OMS, ICROF, Santiago Chile.

${ }^{3}$ DDS, Residente Cirugía MáxiloFacial CAS-UDD, Santiago Chile

${ }^{4}$ DDS, Residente Cirugía Máxilofacial UNAB, Santiago Chile.

${ }^{5}$ DDS, Santiago Chile

Received: 2020-11-09 Accepted: 2021-02-17 
La literatura de hoy en día nos entrega otras alternativas de tratamiento para la reconstrucción de defectos mandibulares, una de esas opciones es el injerto óseo no vascularizado, que puede obtenerse de diferentes zonas, tales como: cresta ilíaca, peroné, calota, radial, etc. Entre las ventajas de esta técnica, destacan un menor tiempo operatorio y disminución de la morbilidad quirúrgica (Osborn et al., 2018) en comparación con la granmayoría de injertos microvascularizados que requieren de una paleta osteomiocutanea para dicha reconstrucción. Por lo tanto los injertos no vascularizados tienen menor invasividad quirúrgica en los pacientes pediátricos y sumado a lo anterior, se debe mencionar la gran capacidad de reparación y regeneración que poseen los niños, que convierten este tipo de injerto en una excelente alternativa terapéutica.

Según la clasificación de tumores de cabeza y cuello de la Organización Mundial de la Salud 2017, dentro de los tumores odontogénicos, el Ameloblastoma es divido en 3 entidades diagnósticas: uniquístico, periférico y metastásico. A su vez, según su histología se clasifica en folicular, plexiforme, acantomatoso y desmoblastico.

El Ameloblastoma es considerado dentro del grupo de tumores benignos de origen odontogénico epitelial. Se presenta generalmente asintomático (de hallazgo radiográfico), y tiene un gran potencial de agresividad (Hendra et al., 2020).

El propósito de este reporte es presentar el caso de un paciente pediátrico con el diagnóstico de ameloblastoma de cuerpo y rama mandibular derecho, tratado vía cirugía resectiva, más injerto libre no vascularizado de cresta iliaca.

\section{REPORTE DE CASO}

Paciente sexo masculino 11 años de edad con antecedente de aumento de volumen mandibular derecho. Fue derivado a evaluación en práctica privada, ICROF (instituto de cirugía y rehabilitación oro facial), Santiago de Chile. El paciente consulta con una ortopantomografía donde se visualiza una zona radiolúcida multilocular extensa en región de cuerpo y rama mandibular derecha, con desplazamiento de piezas dentarias. No presentaba antecedentes mórbidos personales o familiares relevantes, en condición clínica asintomático y con dentición permanente.
Se solicitó tomografía Cone Beam como examen imagenológico complementario, en el cuál se visualiza una zona hipodensa extensa que compromete cuerpo y rama mandibular subcondílea con expansión de tablas vestibular, lingual y perforación de la cortical vestibular (Fig. 1). Se realizó inicialmente una cirugía de biopsia bajo anestesia local resultando el informe del estudio histopatológico Ameloblastoma tipo histológico Folicular.
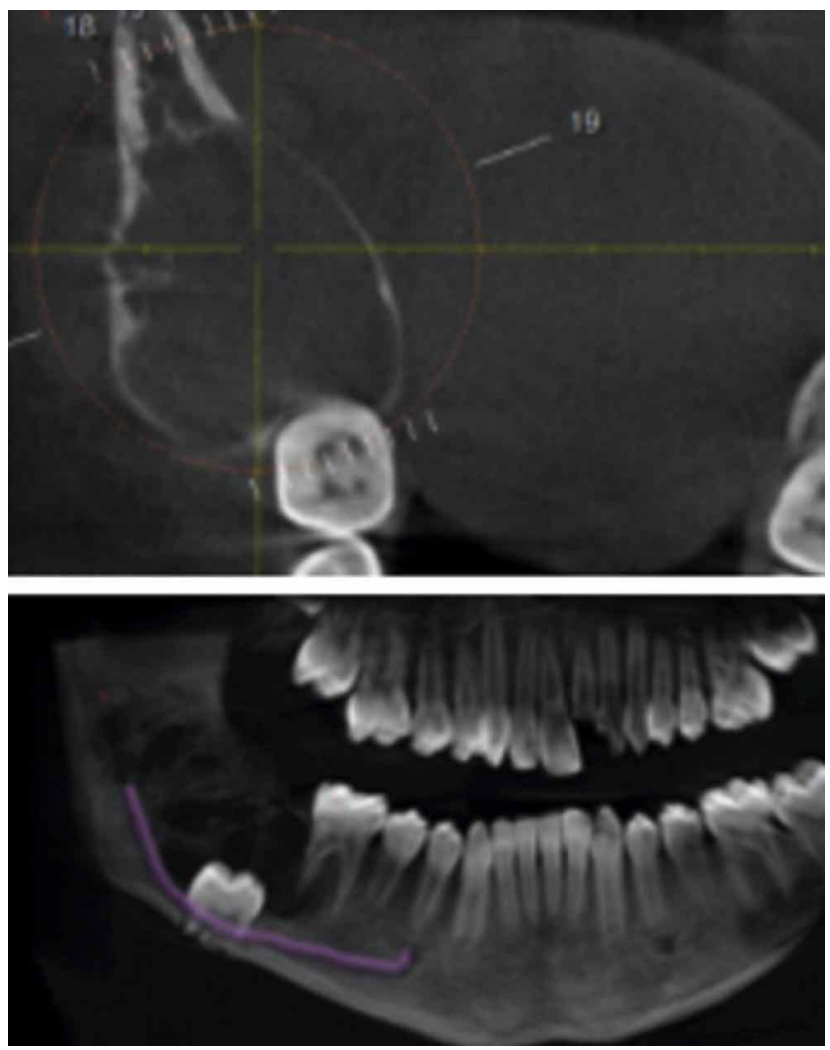

Fig. 1. Imagen preoperatoria de corte axial y vista panorámica 3D de cone beam.

El propósito de la intervención posterior al resultado del estudio histopatológico fue una cirugía resectiva bajo anestesia general, con margen de seguridad de $8 \mathrm{~mm}$ en cada extremo y reconstrucción del defecto con injerto óseo autólogo no microvascularizado de cresta iliaca anterior (Fig. 2).

El injerto se posicionó con una placa de reconstrucción 2.4 previamente adaptada en modelo estereolitográfico 3D, y se realizó cobertura del injerto con hemoderivados de plasma rico en fibrina. La pieza quirúrgica fue enviada a un nuevo estudio histopatológico que confirmó el diagnostico inicial. 

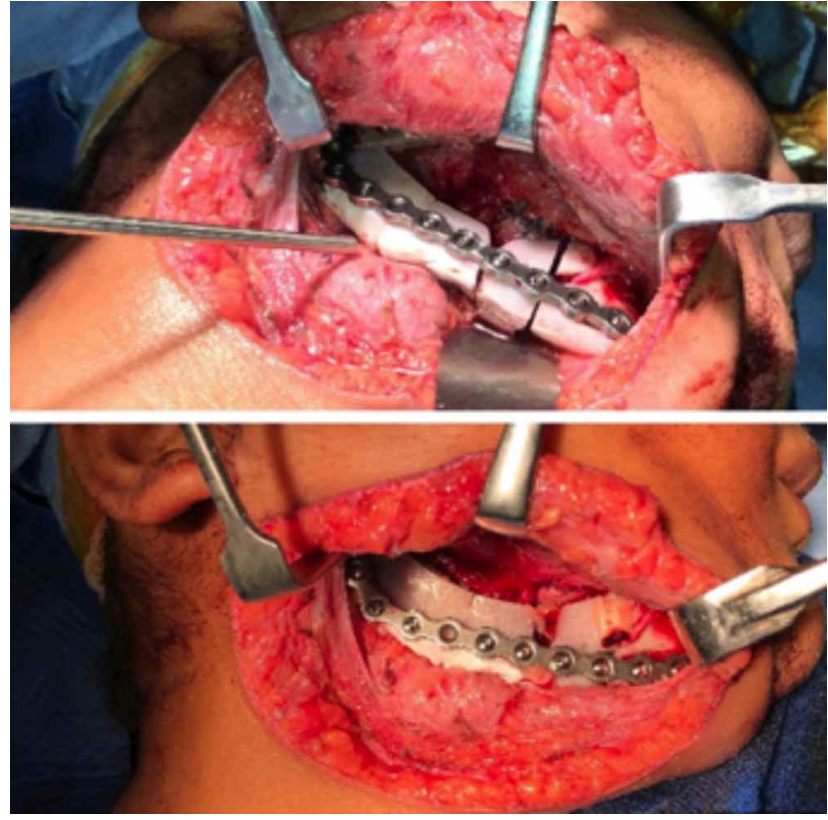

Fig. 2. Imagen intraoperatoria de injerto óseo autogeno no vascularizado seccionado, adaptado y fijado con placa de reconstrucción 2.4 .

Se hizo un seguimiento clínico y radiográfico a los 2, 4, 6 y 12 meses post cirugía sin presentar complicaciones o recidivas, verificando en conebeam (8 meses post cirugía; Fig. 3), neoformación ósea com-
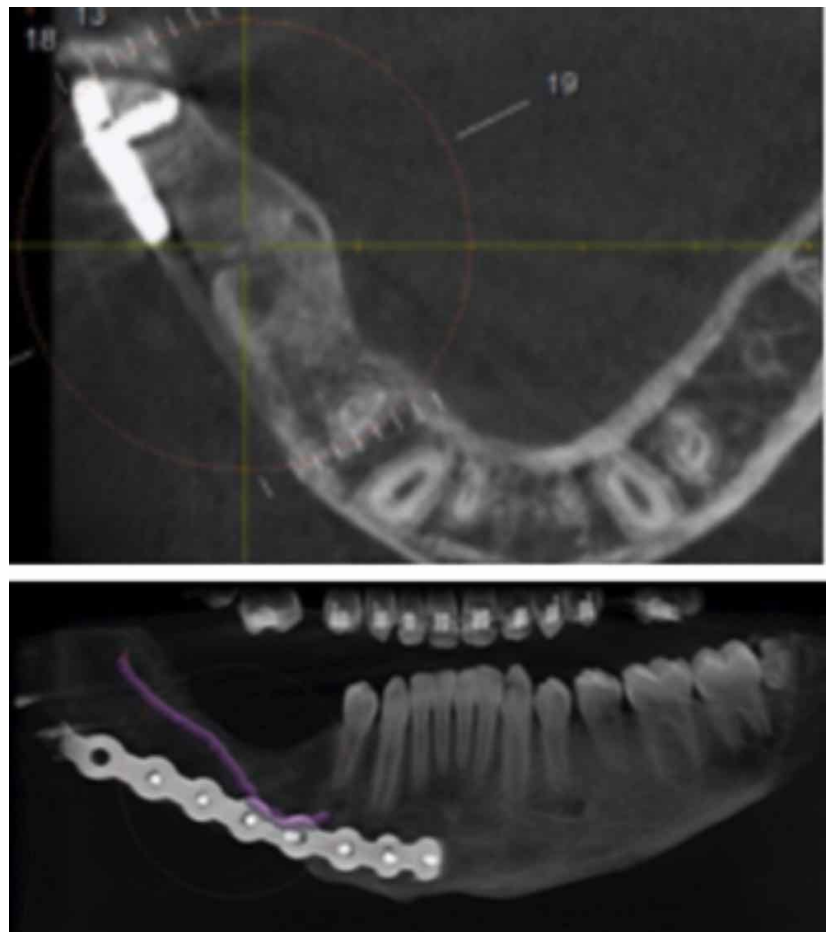

Fig. 3. Imagen de corte axial y vista panorámica 3D de cone beam 8 meses post cirugía. pleta de los segmentos de cuerpo y rama mandibular reconstruidos (Fig. 4).

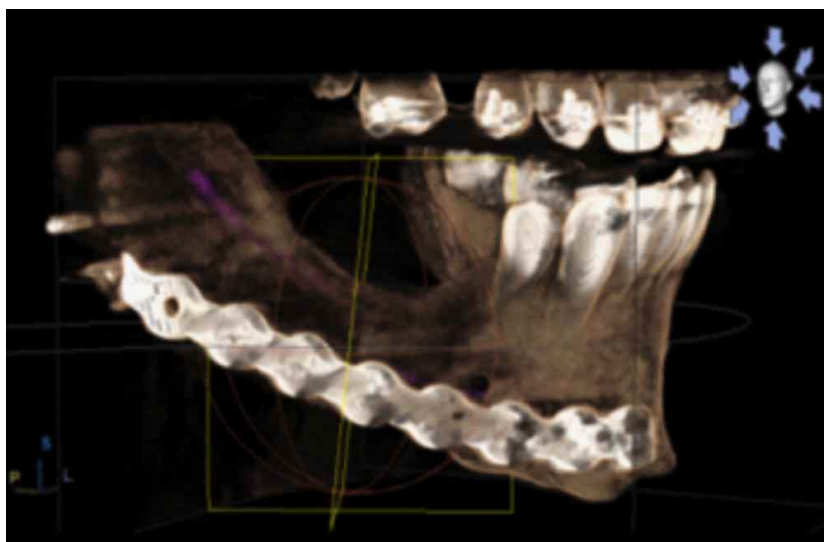

Fig. 4. Imagen postoperatoria, reconstrucción 3D de cuerpo y ángulo mandibular derecho, 8 meses post cirugía.

El paciente se mantiene en control constante por parte del equipo de Cirugía Maxilofacial y continua en tratamiento interdisciplinario con Ortodoncia, Odontopediatría, Kinesiología y Fonoaudiología.

La obtención de estos resultados permite aportar evidencia para el uso de injertos no vascularizados como alternativa de tratamiento en reconstrucción de defectos óseos mandibulares extensos de pacientes pediátricos.

\section{DISCUSIÓN}

La reconstrucción mandibular en pacientes pediátricos es compleja y controversial, pero se recomienda realizar en el mismo acto quirúrgico resectivo, permitiendo de este modo mantener y recuperar funciones orofaciales, estéticas y psicosociales del paciente.

La principal controversia en cirugía reconstructiva de pacientes pediátricos, tiene que ver con el crecimiento y desarrollo del esqueleto facial y del sitio donante. Estudios cefalométricos demuestran que el crecimiento mandibular del hombre se completa entre los $14-16$ años. En este tipo de desarrollo, el cóndilo y sínfisis mandibular son centros de crecimiento fundamentales junto a procesos de reabsorción-aposición ósea, desarrollo de dientes, entre otros (Costello et al., 2012).

A principios de la última década, los estudios relacionados con el uso de injerto no vascularizado de cresta ilíaca, en reconstrucción mandibular inmedia- 
TAPIA, C. P.; ARRUÉ, D. P.; MORDOH, C. S.; REYES, A. C. \& ROSENBERG, D. B. Neo formación ósea de rama y cuerpo mandibular tras uso de injerto no vascularizado de cresta ilíaca en reconstrucción inmediata tras resección de Ameloblastoma Folicular en paciente de 11 años. Caso clínico. Int. J. Odontostomat., 15(3):569-573, 2021.

ta, siguen validando este tratamiento para resecciones menores de $5-6 \mathrm{~cm}$ y de preferencia en pacientes no irradiados (Handschel et al., 2011). Respecto a las longitudes máximas como riesgo de fracaso, se empieza a cuestionar el dogma de la necesidad de injertos no vascularizados en reconstrucciones de más de $6 \mathrm{~cm}$ de longitud, considerando la posible pérdida de vascularización en una extensión muy amplia y que adicionalmente deben ser seccionados para su adaptación (Schlieve et al.). Ejemplo de esto es una presentación de Marechek et al, de 29 pacientes bajo reconstrucción mandibular (con 22 defectos mayores a $6 \mathrm{~cm}$ ), donde el $91 \%$ de los casos tuvo éxito quirúrgico (Marechek et al., 2019).

Existen controversias respecto al tratamiento del ameloblastoma en niños debido a dos factores: 1. El crecimiento facial continuo y fisiología ósea (hueso más esponjoso, aumento del recambio óseo y periostio reactivo); 2. La presencia de dientes no erupcionados.

Zhang et al. (2010) en su estudio retrospectivo muestra que todos los pacientes (37) fueron manejados quirúrgicamente; Ocho pacientes $(21,6 \%, 6$ ameloblastoma sólidos y 2 de tipo uniquístico) fueron tratados con resección aplicando margen de seguridad (segmentaria o hemi-mandibulectomía y reconstrucción con injerto óseo de cresta iliaca) y no se describen recurrencias locales (Zhang et al.).

Respecto al análisis anterior, Au et al., evidenció un aumento significativo en la recidiva del ameloblastoma tratado con enucleación en comparación a terapias de resección marginal; las técnicas quirúrgicas conservadoras muchas veces exponen al paciente a la recidiva de esta patología o al desarrollo de entidades malignas como el carcinoma ameloblástico (Au et al., 2019).

Existen bases científicas para plantear el uso de injertos no vascularizados de cresta ilíaca en reconstrucciones mandibulares inmediatas de defectos óseos menores a $6 \mathrm{~cm}$ en pacientes pediátricos. Nuestro reporte de muestra éxito con neoformación ósea completa de cuerpo y rama mandibular en un defecto óseo mayor a $10 \mathrm{~cm}$, lo que se contrapone favorablemente con el reporte de la literatura en niños y adultos. En este caso el hecho de realizar el tratamiento resectivo con injerto óseo no microvascularizado sin recidiva, logrando regeneración ósea en un paciente de once años y seguimiento clínico-radiográfico de un año, muestra indicio de que la técnica reconstructiva es efectiva en defectos óseos mayores a $6 \mathrm{~cm}$ (subcondilares) con mínina afección respecto al desarrollo óseo del paciente pediátrico.

TAPIA, C. P.; ARRUÉ, D. P.; MORDOH, C. S.; REYES, A. C. \& ROSENBERG, D. B. Neo bone formation of the mandibular branch and body after the use of a nonvascularized iliac crest graft in immediate reconstruction after resection of follicular ameloblastoma in an 11-yearold patient. Clinical case. Int. J. Odontostomat., 15(3):569573, 2021.

ABSTRACT: The gold standard for aggressive mandibular ameloblastomas, with exeresis margins higher than $6 \mathrm{~cm}$, is immediate resection and reconstruction with autologous microvascularized bone graft. A controversy arises because the main reason to endorse this treatment relies on the blood supply for wide extensions of implanted bone. It is necessary in these cases to use a correct stabilization and attachment of the graft employing titanium reconstruction plates and to verify the absence of tissue undergoing radiotherapy. All of the above-mentioned elements are relevant for surgical success. In pediatric patients, the osseointegration and bone repair procedures benefit from physiological growth that entails the presence of multiple growth factors. The aim of this case report is to describe the management of mandibular ameloblastoma diagnosed in a pediatric patient and treated by resection and reconstruction with a non-vascularized $10 \mathrm{~cm}$ iliac crest graft thus achieving 3D bone neoformation of the reconstructed area.

KEY WORDS: pediatrics, autogenous
nonvascularized bone graft, mandibular
reconstruction.

\section{REFERENCIAS BIBLIOGRÁFICAS}

Akinbami, B. O. Reconstruction of continuity defects of the mandible with non-vascularized bone grafts. Systematic literature review. Craniomaxillofac. Trauma Reconstr., 9(3):195-205, 2016.

Schlieve, T.; Hull, W.; Miloro, M. \& Kolokythas, A. Is immediate reconstruction of the mandible with nonvascularized bone graft following resection of benign pathology a viable treatment option?. J. Oral Maxillofac. Surg., 73(3):541-9, 2015.

Okoturo, E. Non-vascularised iliac crest bone graft for immediate reconstruction of lateral mandibular defect. Oral Maxillofac. Surg., 20(4):425-9, 2016.

Osborn, T. M.; Helal, D. \& Mehra, P. lliac crest bone grafting for mandibular reconstruction: 10-year experience outcomes. J. Oral Biol. Craniofac. Res., 8(1):25-9, 2018.

Hendra, F. N.; Van Cann, E. M.; Helder, M. N.; Ruslin, M.; de Visscher, J. G.; Forouzanfar, T. \& de Vet, H. C. W. Global incidence and profile of ameloblastoma: A systematic review and metaanalysis. Oral Dis., 26(1):12-21, 2020.

Costello, B. J.; Rivera, R. D.; Shand, J. \& Mooney, M. Growth and development considerations for craniomaxillofacial surgery. Oral Maxillofac. Surg. Clin. North Am., 24(3):377-96, 2012. 
TAPIA, C. P.; ARRUÉ, D. P.; MORDOH, C. S.; REYES, A. C. \& ROSENBERG, D. B. Neo formación ósea de rama y cuerpo mandibular tras uso de injerto no vascularizado de cresta ilíaca en reconstrucción inmediata tras resección de Ameloblastoma Folicular en paciente de 11 años. Caso clínico. Int. J. Odontostomat., 15(3):569-573, 2021.

Handschel, J.; Hassanyar, H.; Depprich, R. A.; Ommerborn, M. A.; Sproll, K. C.; Hofer, M.; Kübler, N. R. \& Naujoks, C. Nonvascularized iliac bone grafts for mandibular reconstruction-requirements and limitations. In Vivo, 25(5):795-9, 2011.

Marechek, A.; AlShare, A.; Pack, S.; Demko, C.; Quereshy, F. A. \& Baur, D. Nonvascularized bone grafts for reconstruction of segmental mandibular defects: is length of graft a factor of success?. J. Oral Maxillofac. Surg., 77(12):2557-66, 2019.

Zhang, J.; Gu, Z.; Jiang, L.; Zhao, J.; Tian, M.; Zhou, J. \& Duan, Y. Ameloblastoma in children and adolescents. Br. J. Oral Maxillofac. Surg., 48(7):549-54, 2010.

Au, S. W.; Li, K. Y.; Choi, W. S. \& Su, Y. X. Risk factors for recurrence of ameloblastoma: a long-term follow-up retrospective study. Int. J. Oral Maxillofac. Surg.,;48(10):1300-6, 2019.

Dirección para correspondencia:

Benjamín Rosenberg D.

Servicio Cirugía Máxilo Facial Red Salud

Vitacura, Servicio de Cirugía Máxilo Facial HRR

Santiago

CHILE

E-mail: brosenbergd@gmail.com 